\title{
EXPONENTIAL STABILIZATION WITHOUT GEOMETRIC CONTROL
}

\author{
EMmANuEL SCHENCK
}

\begin{abstract}
We present examples of exponential stabilization for the damped wave equation on a compact manifold in situations where the geometric control condition is not satisfied. This follows from a dynamical argument involving a topological pressure on a suitable uncontrolled set.
\end{abstract}

\section{Introduction}

The stabilization problem consist in studying the decay of energy for a dissipative wave equation on a manifold $[3,9,2]$. Originally aimed at controlling the vibrations of large structures, this study has possibly numerous applications, such as for instance sensor and actuators placements in real control problems. Furthermore, such questions always rely on the study of non-selfadjoint operators, the spectrum of which still lacks a good understanding.

A simple model for the stabilization problem is given by the damped wave equation on a compact manifold $(M, g)$ with no boundary. If $a \in C^{\infty}(M)$, this equation reads

$$
\left(\partial_{t}^{2}-\Delta_{g}+2 a \partial_{t}\right) u=0 .
$$

If $a \geq 0$ is non-identically zero, the energy of the waves, given by

$$
E(u, t)=\frac{1}{2}\left(\|\nabla u\|_{L^{2}(M)}^{2}+\left\|\partial_{t} u\right\|_{L^{2}(M)}^{2}\right),
$$

is decreasing in time, and satisfies $E(u, t) \stackrel{t \rightarrow \infty}{\longrightarrow} 0$. This energy decay, controlled by some specific norm of the initial data $\left.\boldsymbol{u}_{0} \stackrel{\text { def }}{=}\left(u, \partial_{t} u\right)\right|_{t=0}$, is at the center of the stabilization problem. For $s>0$ one look for a function $f_{s}(t) \geq 0$ such that

$$
E(u, t) \leq f_{s}(t)\left\|\boldsymbol{u}_{0}\right\|_{\mathcal{H}^{s}}^{2}, \quad\left\|\boldsymbol{u}_{0}\right\|_{\mathcal{H}^{s}}^{2}=\|u(\cdot, 0)\|_{H^{1+s}(M)}^{2}+\left\|\partial_{t} u(\cdot, 0)\right\|_{H^{s}(M)}^{2} .
$$

A particularly interesting situation is the exponential decay, namely when $f_{s}(t)=$ $C \mathrm{e}^{-\beta_{s} t}$ for some $\beta_{s}>0$ and $C>0$ independant of $u$. The choice of the norm $\|\cdot\|_{\mathcal{H}^{s}}$ is determined by the fact that the most general initial data $\left.\left(u, \partial_{t} u\right)\right|_{t=0}$ for the Cauchy problem arising from (1.1), belong to $\mathcal{H}^{0} \stackrel{\text { def }}{=} H^{1}(M) \times H^{0}(M)$, see below. The case $s>0$ simply express that the energy is controlled "at a greater cost of derivatives" on the initial conditions.

The use of global hypotheses concerning the geometry and dynamics of the problem together with microlocal techniques has allowed a major breakthrough concerning the stabilization problem : in $[13,3]$, it has been established an exponential decay of the

Received by the editors November 28, 2010. 
energy for all initial data, under the hypothesis of geometric control (GCH). This hypothesis can be stated as follows:

There is some $T_{0}>0$ such that every geodesics with length $\geq T_{0}$ meets $\operatorname{supp} a$.

Until now, GCH has been an unavoidable assumption in order to get an exponential decay $[3,8]$. Conversely, the failure of GCH implies that no exponential decay can occur for waves with the most general initial data, i.e. for $s=0$, see [9].

Recently, some efforts has been made to investigate the energy decay when GCH fails. In [5], N. Burq and M. Hitrik have studied the damped wave equation in partially rectangular domains in the plane, a situation that includes for instance the Bunimovich stadium. In this latter case, assuming only $a>0$ in the two half-discs, they were able to show a polynomial decay of the waves : for any $s>0$, they obtained

$$
E(u, t) \leq C_{s}(\log t)^{s+2} t^{-s}\left\|\boldsymbol{u}_{0}\right\|_{H_{0}^{1+s} \times H^{s}}^{2} .
$$

Note that GCH may fail, since some bouncing ball trajectories can stay out of $\operatorname{supp} a$. In an example close to what we will develop further, Christianson [7] obtained a subexponential decay for the damped wave equation on a compact Riemannian manifold, where GCH holds outside a neighbourhood of a closed, hyperbolic orbit. For any $s>0$, he showed that there exists some $C_{s}>0$ such that

$$
E(u, t) \leq C_{s} \mathrm{e}^{-C_{s} \sqrt{t}}\left\|\boldsymbol{u}_{0}\right\|_{\mathcal{H}^{s}}^{2} .
$$

The result we will present here address the following question : are there situations where GCH fails, while exponential stabilization holds ? In a recent work [14], we showed that for manifolds with strictly negative curvature, if GCH is dropped and replaced by an hypothesis involving the topological pressure of the geodesic flow, an exponential decay can happen. However, computing explicitely the topological pressure is, in general, a difficult task, and explicit examples showing the non-equivalence of the control and pressure hypotheses were still lacking. As a consequence of a dynamical result of independent interest (Theorem 5 below), we provide here a concrete example. To the author knowledge, this is the first case where an exponential decay of the energy occurs, while the geometric control condition fails. It can be stated as follows (see also Figure 1.1) :

Theorem 1. Let $(M, g)$ be a closed Riemannian manifold of dimension $d \geq 2$, with negative curvature. Let $\gamma \in S^{*} M$ be a periodic geodesic, $a \in C^{\infty}(M)$ a non-negative function, lifted on $S^{*} M$ so that it is constant in the fibers, and $s>0$ a real number. There exists $\varepsilon>0$ so that if $a$ is vanishing in an $\varepsilon$ neighbourhood of $\gamma$ and $a>0$ outside this neighbourhood, there exists a sequence $\left\{\beta_{n}\right\}_{n \geq 0}$ satisfying $\beta_{n} \stackrel{n \rightarrow \infty}{\longrightarrow}+\infty$ with the following property : if $n$ is sufficiently large, there is $C_{s}>0$ such that for any solution $u$ of $\left(\partial_{t}^{2}-\Delta_{g}+2 \beta_{n} a \partial_{t}\right) u=0$ with initial conditions $\left.\left(u, \partial_{t} u\right)\right|_{t=0}=\boldsymbol{u}_{0}$, we have

$$
E(u, t) \leq C_{s} \mathrm{e}^{-C_{s} t}\left\|\boldsymbol{u}_{0}\right\|_{\mathcal{H}^{s}}^{2} .
$$

For $s>d / 2$, we have a more precise statement : for any $\epsilon>0$,

$$
E(u, t) \leq C_{\epsilon} \mathrm{e}^{-(G-\epsilon) t}\left\|\boldsymbol{u}_{0}\right\|_{\mathcal{H}^{s}}^{2},
$$

where $G>0$ is defined in (1.7). 


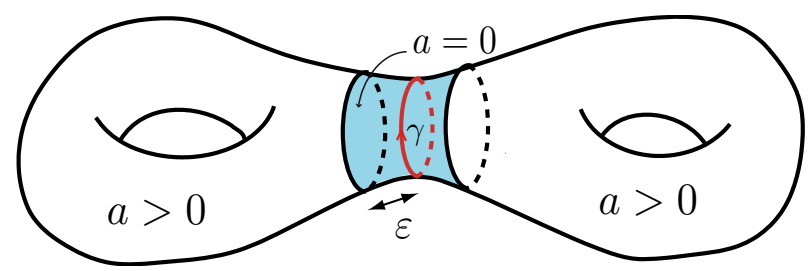

FiguRE 1.1. A simple example where the geometric control hypothesis fails : the closed geodesic $\gamma$ is undamped. However, if $\varepsilon$ is small and the damping $a$ is replaced by $\beta a$ with $\beta>0$ sufficiently large, the energy of the waves decays exponentially fast for any initial data, in $H^{1+s} \times H^{s}, s>0$.

As a remark, we mention that a forthcoming work of Burq and Christianson [6] shows that in some specific cases with only one uncontrolled geodesic, the inequality (1.2) is optimal. In our situation, to get an exponential decay, the global hypothesis about the negative curvature of $M$ plays a crucial role.

1.1. The topological pressure. We will be concerned with a compact Riemannian manifold $(M, g)$ with strictly negative curvature. We call the geodesic flow

$$
\Phi^{t}=\mathrm{e}^{t H_{p}}: T^{*} M \rightarrow T^{*} M
$$

where

$$
p: T^{*} M \ni(x, \xi) \mapsto g_{x}(\xi, \xi)=\|\xi\|_{x}^{2} \in \mathbb{R}
$$

is the Hamiltonian and $H_{p}$ the corresponding Hamilton vector field. Note also that $p$ is the principal symbol of $-\Delta_{g}$. Since $M$ has strictly negative curvature, it is a well known fact that the flow generated by $H_{p}$ on constant energy layers $\mathcal{E}=p^{-1}(E) \subset$ $T^{*} M, E>0$ has the Anosov property, see Appendix A. The topological pressure with respect to the geodesic flow on $\mathcal{E}$ is a functional

$$
\operatorname{Pr}: f \in C^{\infty}(\mathcal{E}) \rightarrow \overline{\mathbb{R}}
$$

which evaluates in some sense the complexity of the flow via the ergodic means of the function $f$. For Anosov flows, a rather simple definition can be given in terms of periodic orbits. A definition suitable for general dynamical systems is given in Appendix A. For $T>0$, let $P(T)$ be the set of closed geodesics with length $\leq T$. Then, one has :

$$
\operatorname{Pr}(f) \stackrel{\text { def }}{=} \lim _{T \rightarrow \infty} \frac{1}{T} \log \left(\sum_{\gamma \in P(T)} \mathrm{e}^{\int_{\gamma} f}\right) .
$$

If $f \equiv 0$, one simply counts in the sum the number of periodic orbit of length $\leq T$, and $\operatorname{Pr}(0)$ is equal to the topological entropy of the flow. From now on, we restrict ourselves to the flow on the unit cotangent bundle $\Phi^{t}: S^{*} M \rightarrow S^{*} M$, where $S^{*} M \subset T^{*} M$ is made of points with norm one covectors :

$$
S^{*} M \stackrel{\text { def }}{=} p^{-1}(1)=\left\{(x, \xi) \in T^{*} M:\|\xi\|_{x}=1\right\} .
$$


For simplicity we will note $X \stackrel{\text { def }}{=} S^{*} M$ in the following. This is a compact, $2 d-1$ dimensional manifold. We will consider it as a Riemannian space too, equipped with a metric coming from $g$ such as in the Sasaki construction.

As shown by Walters in general settings, the topological pressure has deep connections with invariant measures on $X$. A probability measure $\mu$ on $X$ is said to be invariant, if for any Borel subset $B \subset X$ and $t \geq 0$ one has $\mu\left(\Phi^{-t}(B)\right)=\mu(B)$. We will denote by $\mathfrak{M}$ the set of invariant measures on $X$. For instance, the Liouville measure coming from the symplectic structure of $X$ belongs to $\mathfrak{M}$. The variational principle [16] asserts that one can recover the topological pressure from the knowledge of the set $\mathfrak{M}$ :

$$
\operatorname{Pr}(f)=\sup _{\mu \in \mathfrak{M}}\left(h_{K S}(\mu)+\int_{X} f d \mu\right) .
$$

Here, $h_{K S}$ stands for the Kolmogorov-Sinai entropy of $\mu$. In some cases, the sup in the above equation is attained for some probability measure $\mu_{f} \in \mathfrak{M}$ : we say that $\mu_{f}$ is an equilibrium state for the potential $f$. For Anosov diffeomorphisms, such an equilibrium state always exists and is unique.

Below, we will use the notion of topological pressure of functions on sets $K \subset X$ which are compact and invariant under the geodesic flow. Pesin and Pitskel [11] have shown that the pressure of $f$ on $K$, denoted by $\operatorname{Pr}_{K}(f)$, is also given by a variational principle. Denote by $\mathfrak{M}(K) \subset \mathfrak{M}$ the set of invariant measures $\mu$ satisfying $\mu(K)=1$. Then,

$$
\operatorname{Pr}_{K}(f)=\sup _{\mu \in \mathfrak{M}(K)}\left(h_{K S}(\mu)+\int_{K} f d \mu\right) .
$$

1.2. Energy decay under a pressure condition. Before stating our main result, we recast the damped wave equation in a standard way convenient for spectral analysis. With $\boldsymbol{u}=\left(u, \mathrm{i} \partial_{t} u\right)$ one can rewrite (1.1) as

$$
\partial_{t} \boldsymbol{u}=-\mathrm{i} \mathcal{B} \boldsymbol{u}, \quad \mathcal{B}=\left(\begin{array}{cc}
0 & \mathrm{Id} \\
-\Delta_{g} & -2 \mathrm{i} a
\end{array}\right) .
$$

Via the evolution group $\mathrm{e}^{-\mathrm{i} t \mathcal{B}}$, we can identify any solution of (1.5) with its initial data $\boldsymbol{u}_{0} \in H^{1} \times H^{0}$. The metastable modes of the damped wave equation (1.1) can be written in the form $\psi_{n}(t, x)=v(x) \mathrm{e}^{-i t \tau_{n}}$ with $\tau_{n} \in \operatorname{Spec} \mathcal{B}$. The spectrum of $\mathcal{B}$ is a discrete set of complex numbers, localized below the real axis, in the strip $\mathbb{R}+\mathrm{i}[-2 \sup a, 0]$. The only real eigenvalue is $\tau=0$, associated to constant solutions. Let us define the function $a^{u}$ by

$$
a^{u}:\left\{\begin{array}{l}
X \rightarrow X \\
\rho \mapsto-\bar{a}+\frac{1}{2} \log J^{u}
\end{array}\right.
$$

where $J^{u}$ is the unstable Jacobian (see Appendix A), and

$$
\bar{a}: X \ni \rho \mapsto \int_{0}^{1} a \circ \Phi^{t}(\rho) d t .
$$

The function $a$ is considered above as a function on $T^{*} M$, depending only on the position variables. The main results of [14] can be stated as follows : 
Theorem 2. [14] Let $(M, g)$ be a compact Riemannian manifold with negative curvature and no boundary, and $a \in C^{\infty}(M)$ positive, non-identically zero. Suppose that

$$
\operatorname{Pr}\left(a^{u}\right)<0
$$

Then,

$$
G \stackrel{\text { def }}{=} \min \left(\left|\operatorname{Pr}\left(a^{u}\right)\right|, \inf _{\tau \in \operatorname{Spec} \mathcal{B} \backslash\{0\}}|\Im \tau|\right)>0,
$$

namely there is a spectral gap below the real axis. Furthermore, if $s>d / 2$, then for any $\varepsilon>0$ we have

$$
E(u, t) \leq C_{\varepsilon} \mathrm{e}^{-(G-\varepsilon) t}\|\boldsymbol{u}\|_{H^{1+s} \times H^{s}} .
$$

With a standard interpolation argument, the last assertion implies that for any $s>0$, there is some $C_{s}>0$ such that

$$
E(u, t) \leq C_{s} \mathrm{e}^{-C_{s} t}\|\boldsymbol{u}\|_{H^{1+s} \times H^{s}} .
$$

The preceding theorem is proved using methods developed by Anantharaman [1] and Nonnenmacher-Zworski [10]. The condition $\operatorname{Pr}\left(a^{u}\right)<0$ can be compared to the condition in [10] on the topological pressure of the flow on the trapped set, used to prove a gap without resonances below the real axis in the semiclassical limit.

1.3. Main result. Since we are interested in situations where the geometric control does not hold, we have to define an appropriate "undamped" set of $X$. It turns out that the set made of non-controlled geodesics, given by

$$
\mathcal{N} \stackrel{\text { def }}{=} \bigcap_{t \in \mathbb{R}}\left\{\rho \in X: a\left(\Phi^{t}(\rho)\right)=0\right\},
$$

is too large for our purposes : we will rather define a non-controlled subset of $X$ starting from the point of view of the invariant measures $\mathfrak{M}$ of $X$. For this, we introduce the following

Definition 3. An invariant measure $\mu \in \mathfrak{M}$ is minimizing if it satisfies $\int_{X} a d \mu=a_{0}$, where

$$
\min _{\mu \in \mathfrak{M}}\left(\int_{X} a d \mu\right)=a_{0} .
$$

We call $\mathfrak{M}_{0} \subset \mathfrak{M}$ the set of minimizing measures on $M$. In the interesting cases where GCH does not holds, it is clear that $a_{0}=0$. Notice also that $\min _{\mu \in \mathfrak{M}}\left(\int_{X} \bar{a} d \mu\right)=$ $a_{0}$. Let us now define the following set :

$$
\mathcal{K} \stackrel{\text { def }}{=} \bigcup_{\mu \in \mathfrak{M}_{0}} \operatorname{supp} \mu,
$$

which will be our "undamped set". It is clear that $\mathcal{K}$ is a compact, flow-invariant subset of $X$. It has zero Liouville measure, since this measure is ergodic with respect to the geodesic flow in negative curvature. 
Remark 4. One has $\mathcal{K} \subset \mathcal{N}$ (see Lemma 8 below), but $\mathcal{K} \neq \mathcal{N}$ in general. For instance, take $\gamma$ and $\beta$ two geodesics, with $\gamma$ periodic and $\beta$ homoclinic to $\gamma$, which means that any point of $\beta$ belongs at the same time to the stable and the unstable manifold of $\gamma$ (see Appendix A). Finally, chose $a$ positive outside a small enough neighbourhood of $\gamma \cup \beta$, and $a=0$ near $\gamma \cup \beta$. In this case it is easy to see that $\mathcal{N}=\{\gamma \cup \beta\}$, while $\mathcal{K}=\{\gamma\}$.

Our main result, although still quite general, allows to check the hypothesis (1.6) to a pressure condition on $\mathcal{K}$ only, which may be easier to evaluate :

Theorem 5. Let $\bar{a}, a_{0}, J^{u}$ and $\mathcal{K}$ be as above. There is a sequence $\left\{\beta_{n}\right\}_{n \geq 0}$ of positive numbers with $\lim _{n \rightarrow \infty} \beta_{n}=+\infty$ such that

$$
\lim _{n \rightarrow+\infty} \operatorname{Pr}\left(-\beta_{n} \bar{a}+\frac{1}{2} \log J^{u}\right)+\beta_{n} a_{0}=\operatorname{Pr}_{\mathcal{K}}\left(\frac{1}{2} \log J^{u}\right) .
$$

Basically speaking, the preceding result says that if the damping is strong enough, the damped trajectories have a negligible contribution to $\operatorname{Pr}\left(a^{u}\right)$. Remain the undamped ones, for which the pressure of $a^{u}$ reduce to the right hand side of (1.11). From this purely dynamical result, we immediately deduce the following application:

Corollary 6. Suppose that $\operatorname{Pr}_{\mathcal{K}}\left(\frac{1}{2} \log J^{u}\right)<0$. Then, for some large enough $\beta>0$, we have $\operatorname{Pr}\left(-\beta a+\frac{1}{2} \log J^{u}\right)<0$. It follows that (1.8), (1.9) hold.

Remark 7 . The following question, to which we are unable to answer in full generality at this point, would have interesting consequences :

Question : is $\mathcal{K}$ a locally maximal hyperbolic subset of $X$ ?

Indeed, the hypothesis $\operatorname{Pr}_{\mathcal{K}}\left(\frac{1}{2} \log J^{u}\right)<0$ would be in this case related to the size of $\mathcal{K}$ : a "filamentary" set $\mathcal{K}$ is more likely to satisfy $\operatorname{Pr}_{\mathcal{K}}\left(\frac{1}{2} \log J^{u}\right)<0$. For instance, if $d=2$ and if $\mathcal{K}$ is a locally maximal hyperbolic set, i.e. there is a neighbourhood $U$ of $\mathcal{K}$ such that

$$
\mathcal{K}=\bigcap_{t \in \mathbb{R}} \Phi^{t}(U),
$$

then there is a direct relationship between the Hausdorff dimension of $\mathcal{K}$ and the pressure $\operatorname{Pr}_{\mathcal{K}}\left(\frac{1}{2} \log J^{u}\right)$. More precisely [12],

$$
d_{H}(\mathcal{K})<2 \Longleftrightarrow \operatorname{Pr}_{\mathcal{K}}\left(\frac{1}{2} \log J^{u}\right)<0 .
$$

Hence a positive answer to the above question would simply mean that in dimension 2, checking the Hausdorff dimension of $\mathcal{K}$ (or $\mathcal{N} \supset \mathcal{K}$ ) would be sufficient to decide wether exponential stabilization can occur or not, provided some strong enough damping is applied (note however that it is well known that the set $\mathcal{N}$ in Remark 4 is not locally maximal). For instance, the above remarks imply directly Theorem 1 for $d=2$ once it is checked that $\mathcal{K}=\{\gamma\}$, which is indeed locally maximal. Finally, in higher dimension, there is no obvious link between the Hausdorff dimension $d_{H}(\mathcal{K})$ and the pressure $\operatorname{Pr}_{\mathcal{K}}\left(\frac{1}{2} \log J^{u}\right)$, unless some assumptions of conformality of the flow in the stable and unstable directions are made [12]. 


\section{Proof of Theorem 5}

We are interested in the thermodynamic limit, namely we want to study

$$
\operatorname{Pr}\left(-\beta \bar{a}+\frac{1}{2} \log J^{u}\right) \quad \text { in the limit } \beta \rightarrow+\infty .
$$

The variational principle will be the main tool used to get Theorem 5 . We begin with two simple lemmas.

Lemma 8. The function a satisfies

$$
\left.a\right|_{\mathcal{K}}=a_{0} .
$$

Proof. Without loss of generality we can assume that $a_{0}=0$. Arguing by contradiction, suppose otherwise that there is some $\rho_{0} \in \mathcal{K}$ such that $a\left(\rho_{0}\right)>0$. By continuity, we can find $\epsilon \geq 0$ such that $a>0$ in some Borel subset $B\left(\rho_{0}, \epsilon\right)$ of diameter $\epsilon$ satisfying $B\left(\rho_{0}, \epsilon\right) \subset \operatorname{supp} \mu_{0}$ for some $\mu_{0} \in \mathfrak{M}_{0}$. But then,

$$
\int_{B\left(\rho_{0}, \epsilon\right)} a d \mu_{0}>\mu_{0}\left(B\left(\rho_{0}, \epsilon\right)\right) \inf _{B\left(\rho_{0}, \epsilon\right)} a>0,
$$

which is a contradiction since $\mu_{0}$ is minimizing. In particular, we deduce immediately from the flow invariance of $\mathcal{K}$ that $\mathcal{K} \subset \mathcal{N}$.

Lemma 9. We have $\mathfrak{M}(\mathcal{K})=\mathfrak{M}_{0}$. It follows that for any $f \in C^{\infty}(X)$,

$$
\operatorname{Pr}_{\mathcal{K}}(f)=\sup _{\mathfrak{M}_{0}}\left(h_{K S}(\mu)+\int_{X} f d \mu\right) .
$$

Proof. If $\mu \in \mathfrak{M}(\mathcal{K})$, then $\mu$ is invariant and satisfies $\operatorname{supp} \mu \subset \mathcal{K}$. But from Lemma 8 , we know that $\int_{X} a d \mu=a_{0}$, which precisely means that $\mu$ is minimizing. The inclusion $\mathfrak{M}_{0} \subset \mathfrak{M}(\mathcal{K})$ is obvious, and the last assertion comes from (1.4).

We now prove Theorem 5 . By weak-* compactness, take a sequence $\left(\mu_{\beta_{n}}\right)_{n \geq 0}$ of equilibrium states for the potential $-\beta_{n} \bar{a}+\frac{1}{2} \log J^{u}$ that converges to some limit $\mu_{\infty}$ when $\beta_{n} \stackrel{n \rightarrow \infty}{\longrightarrow} \infty$. We first show that $\mu_{\infty}$ is minimizing. The variational principle states that

$$
\begin{aligned}
\operatorname{Pr}\left(-\beta_{n} \bar{a}+\frac{1}{2} \log J^{u}\right) & =\max _{\mu \in \mathfrak{M}}\left(h_{K S}(\mu)-\beta_{n} \int_{X} \bar{a} d \mu+\frac{1}{2} \int_{X} \log J^{u} d \mu\right) \\
& \leq-\beta_{n} a_{0}+\max _{\mu \in \mathfrak{M}}\left(h_{K S}(\mu)+\frac{1}{2} \int_{X} \log J^{u} d \mu\right) \\
& \leq-\beta_{n} a_{0}+\operatorname{Pr}\left(\frac{1}{2} \log J^{u}\right) .
\end{aligned}
$$

On the other hand, we have

$$
\begin{aligned}
\operatorname{Pr}\left(-\beta_{n} \bar{a}+\frac{1}{2} \log J^{u}\right) & =\max _{\mu \in \mathfrak{M}}\left(h_{K S}(\mu)-\int_{X}\left(\beta_{n} \bar{a}-\frac{1}{2} \log J^{u}\right) d \mu\right) \\
& \geq \max _{\mu \in \mathfrak{M}_{0}}\left(h_{K S}(\mu)-\int_{X}\left(\beta_{n} \bar{a}-\frac{1}{2} \log J^{u}\right) d \mu\right) \\
& \geq-\beta_{n} a_{0}+\operatorname{Pr}_{\mathcal{K}}\left(\frac{1}{2} \log J^{u}\right),
\end{aligned}
$$


where we have used the fact that $\int_{X} \bar{a} d \mu=a_{0}$ for $\mu \in \mathfrak{M}_{0}$ and Lemma 9. From the above inequalities we keep in mind that

$$
\operatorname{Pr}_{\mathcal{K}}\left(\frac{1}{2} \log J^{u}\right) \leq \operatorname{Pr}\left(-\beta_{n} \bar{a}+\frac{1}{2} \log J^{u}\right)+\beta_{n} a_{0} \leq \operatorname{Pr}\left(\frac{1}{2} \log J^{u}\right),
$$

and dividing the preceding equation by $\beta_{n}$, we obtain

$$
\frac{1}{\beta_{n}} \operatorname{Pr}_{\mathcal{K}}\left(\frac{1}{2} \log J^{u}\right) \leq \frac{2 h_{K S}\left(\mu_{\beta_{n}}\right)+\int_{X} \log J^{u} d \mu_{\beta_{n}}}{2 \beta_{n}}+a_{0}-\int_{X} \bar{a} d \mu_{\beta_{n}} \leq \frac{1}{\beta_{n}} \operatorname{Pr}\left(\frac{1}{2} \log J^{u}\right) .
$$

Letting $n \rightarrow \infty$ in the above equation shows that $\int_{X} \bar{a} d \mu_{\beta_{n}} \stackrel{n \rightarrow \infty}{\longrightarrow} a_{0}$, namely $\mu_{\infty}$ is minimizing.

From (2.1), we have the correct lower bound in view of Theorem 5, i.e.

$$
\lim _{n \rightarrow \infty} \operatorname{Pr}\left(-\beta_{n} \bar{a}+\frac{1}{2} \log J^{u}\right)+\beta_{n} a_{0} \geq \operatorname{Pr}_{\mathcal{K}}\left(\frac{1}{2} \log J^{u}\right),
$$

but it remains to show the upper bound. Using the variational principle again, we have

$$
\begin{gathered}
\operatorname{Pr}\left(-\beta_{n} \bar{a}+\frac{1}{2} \log J^{u}\right)+\beta_{n} a_{0}=h_{K S}\left(\mu_{\beta_{n}}\right)-\beta_{n} \int_{X}\left(\bar{a}-a_{0}\right) d \mu_{\beta_{n}}+\frac{1}{2} \int_{X} \log J^{u} d \mu_{\beta_{n}} \\
\leq \quad h_{K S}\left(\mu_{\beta_{n}}\right)+\frac{1}{2} \int_{X} \log J^{u} d \mu_{\beta_{n}} .
\end{gathered}
$$

We know that $\mu_{\beta_{n}} \stackrel{n \rightarrow \infty}{\longrightarrow} \mu_{\infty}$, but the upper semi-continuity of the Kolmogorov-Sinai entropy only allows to deduce that $\lim _{n \rightarrow \infty} h_{K S}\left(\mu_{\beta_{n}}\right) \leq h_{K S}\left(\mu_{\infty}\right)$. This means that

$$
\begin{aligned}
\lim _{\beta_{n} \rightarrow \infty} \operatorname{Pr}\left(-\beta_{n} \bar{a}+\frac{1}{2} \log J^{u}\right)+\beta_{n} a_{0} & \leq h_{K S}\left(\mu_{\infty}\right)+\frac{1}{2} \int_{X} \log J^{u} d \mu_{\infty} \\
& \leq \max _{\mu \in \mathfrak{M}_{0}}\left(h_{K S}(\mu)+\frac{1}{2} \int_{X} \log J^{u} d \mu\right) \\
& \leq \operatorname{Pr}_{\mathcal{K}}\left(\frac{1}{2} \log J^{u} d \mu\right),
\end{aligned}
$$

and this concludes the proof of Theorem 5 .

\section{Application to the damped wave equation}

Although the Corollary 6 is immediate once the limit (1.11) is proved, we describe now how to obtain Theorem 1. We use the notations of the theorem, and show that if $\varepsilon>0$ is small enough, then $\mathcal{K}=\{\gamma\}$. To see this, suppose that there is some $\rho \in \mathcal{V} \backslash\{\gamma\}$ such that for any $t \in \mathbb{R}, \Phi^{t}(\rho) \in \mathcal{V}$. Considering a Poincaré section $S_{0}$ of the flow near $\rho_{0} \in \gamma$, we get that under the first return map $\kappa: S_{0} \rightarrow S_{0}$ the inequality $d\left(\kappa^{n}(\rho), \rho_{0}\right) \leq \varepsilon$ holds for any $n \in \mathbb{Z}$. But $\kappa$ being hyperbolic, it is expanding (see [4] for instance), therefore we know the existence of an $\varepsilon_{0}>0$ such that the only points $\rho \in S_{0}$ which satisfy $d\left(\kappa^{n}(\rho), \rho_{0}\right) \leq \varepsilon_{0}$ for all $n \in \mathbb{Z}$ actually satisfy $\rho=\rho_{0}$. Hence if we take $\varepsilon \leq \varepsilon_{0}$, it follows that $\mathcal{K}=\{\gamma\}$.

Let us now prove that $\operatorname{Pr}_{\gamma}\left(\frac{1}{2} \log J^{u}\right)<0$. This is can be quickly seen from the variational principle. Observe first that $\mathfrak{M}(\gamma)=\mu_{\gamma}$ where $\mu_{\gamma}$ denotes the invariant measure supported on $\gamma$. Using the property (A.1), this implies that

$$
\operatorname{Pr}_{\gamma}\left(\frac{1}{2} \log J^{u}\right)=\int_{\gamma} \frac{1}{2} \log J^{u} d \mu_{\gamma} \leq-(d-1) \lambda<0,
$$


since $h_{K S}\left(\mu_{\gamma}\right)=0$ and in the adapted metric, $J^{u}(\rho) \leq \mathrm{e}^{-(d-1) \lambda}$. This concludes the proof of Theorem 1: for $\beta$ sufficiently large, $\operatorname{Pr}\left(-\beta a+\frac{1}{2} \log J^{u}\right)$ is close enough to $\operatorname{Pr}_{\gamma}\left(\frac{1}{2} \log J^{u}\right)<0$ to be negative.

\section{Appendix A. Negative curvature, Anosov flows and topological pressure}

A.1. Anosov flows. In this paragraph we give some further information concerning the Anosov property of the geodesic flow in negative curvature. An Anosov flow is a flow everywhere hyperbolic, and enjoys the following properties. We denote as above $\mathcal{E}=p^{-1}(E) \subset T^{*} M, E>0$ an energy layer. For any $\rho \in \mathcal{E}$, the tangent space $T_{\rho} \mathcal{E}$ splits into flow, stable and unstable subspaces

$$
T_{\rho} \mathcal{E}=\mathbb{R} H_{p} \oplus E^{S}(\rho) \oplus E^{u}(\rho) .
$$

The spaces $E^{s}(\rho)$ and $E^{u}(\rho)$ are $d-1$ dimensional, and are preserved under the flow map:

$$
\forall t \in \mathbb{R}, \quad d \Phi_{\rho}^{t}\left(E^{s}(\rho)\right)=E^{s}\left(\Phi^{t}(\rho)\right), \quad d \Phi_{\rho}^{t}\left(E^{u}(\rho)\right)=E^{u}\left(\Phi^{t}(\rho)\right) .
$$

Moreover, there exist $C, \lambda>0$ such that

$$
\begin{aligned}
\text { i) } & \left\|d \Phi_{\rho}^{t}(v)\right\| \leq C \mathrm{e}^{-\lambda t}\|v\|, \quad \text { for all } v \in E^{s}(\rho), t \geq 0 \\
\text { ii) } & \left\|d \Phi_{\rho}^{-t}(v)\right\| \leq C \mathrm{e}^{-\lambda t}\|v\|, \quad \text { for all } v \in E^{u}(\rho), t \geq 0 .
\end{aligned}
$$

One can show that there exist a metric on $T^{*} M$ call the adapted metric, for which one can takes $C=1$ in the preceding equations. At each point $\rho$, the spaces $E^{u}(\rho)$ are tangent to the unstable manifold $W^{u}(\rho)$, the set of points $\rho^{u} \in \mathcal{E}$ such that $d\left(\Phi^{t}\left(\rho^{u}\right), \Phi^{t}(\rho)\right) \stackrel{t \rightarrow-\infty}{\longrightarrow} 0$ where $d$ is the distance induced from the adapted metric. Similarly, $E^{s}(\rho)$ is tangent to the stable manifold $W^{s}(\rho)$, the set of points $\rho^{s}$ such that $d\left(\Phi^{t}\left(\rho^{s}\right), \Phi^{t}(\rho)\right) \stackrel{t \rightarrow+\infty}{\longrightarrow} 0$.

The above properties allow us to define now properly the unstable Jacobian. The adapted metric on $T^{*} M$ induces a the volume form $\Omega_{\rho}$ on any $d$ dimensional subspace of $T\left(T_{\rho}^{*} M\right)$. Using $\Omega_{\rho}$, we can define the unstable Jacobian at $\rho$ for time $t$. Let us define the weak-stable and weak-unstable subspaces at $\rho$ by

$$
E^{s, 0}(\rho)=E^{s}(\rho) \oplus \mathbb{R} H_{p}, \quad E^{u, 0}(\rho)=E^{u}(\rho) \oplus \mathbb{R} H_{p} .
$$

We set

$$
J_{t}^{u}(\rho)=\left.\operatorname{det} d \Phi^{-t}\right|_{E^{u, 0}\left(\Phi^{t}(\rho)\right)}=\frac{\Omega_{\rho}\left(d \Phi^{-t} v_{1} \wedge \cdots \wedge d \Phi^{-t} v_{d}\right)}{\Omega_{\Phi^{t}(\rho)}\left(v_{1} \wedge \cdots \wedge v_{d}\right)}, \quad J^{u}(\rho) \stackrel{\text { def }}{=} J_{1}^{u}(\rho),
$$

where $\left(v_{1}, \ldots, v_{d}\right)$ can be any basis of $E^{u, 0}\left(\Phi^{t}(\rho)\right)$. While we do not necessarily have $J^{u}(\rho)<1$, it is true that $J_{t}^{u}(\rho)$ decays exponentially as $t \rightarrow+\infty$.

A.2. Topological pressure : general definition. Let $X$ be a metric space, and $\Phi^{t}: X \rightarrow X$ a continuous one parameter flow. We give here the definition of the topological pressure stated with Bowen balls, although other equivalent definitions are possible, for instance using open covers of $X$ (see [15] for general properties about the topological pressure). For every $\varepsilon>0$ and $T>0$, a set $\mathcal{S} \subset X$ is $(\varepsilon, T)$-separated 
if $\rho, \theta \in \mathcal{S}$ implies that $d\left(\Phi^{t} \rho, \Phi^{t} \theta\right)>\varepsilon$ for some $t \in[0, T]$, where $d$ is the distance on $X$. For $f$ continuous on $X$, set

$$
Z(f, T, \varepsilon)=\sup _{\mathcal{S}}\left\{\sum_{\rho \in \mathcal{S}} \exp \sum_{k=0}^{T-1} f \circ \Phi^{k}(x)\right\} .
$$

The topological pressure $\operatorname{Pr}(f)$ of the function $f$ with respect to the flow $\Phi$ is defined by

$$
\operatorname{Pr}(f)=\lim _{\varepsilon \rightarrow 0} \limsup _{T \rightarrow \infty} \frac{1}{T} \log Z(f, T, \varepsilon) .
$$

\section{Acknowledgements}

It is a pleasure to thank Stéphane Nonnenmacher and Philippe Thieullen for stimulating discussions which are at the origin of this work.

\section{References}

[1] N. Anantharaman, Entropy and the localization of eigenfunctions, Ann. of Math. 168 (2008), no. $2,435-475$.

[2] M. Asch and G. Lebeau, The spectrum of the damped wave operator for a bounded domain in $\mathbb{R}^{2}$, Experimental math. 12 (2003) 227-241.

[3] C. Bardos, G. Lebeau, and J. Rauch, Sharp sufficient conditions for the observation, control and stabilization of waves from the boundary, SIAM J. Control and Optimization 30 (1992), no. $5,1024-1065$.

[4] R. Bowen, Equilibrium states and the ergodic theory of Anosov diffeomorphisms, Vol. 470 of Lect. Notes in Math., Springer (1975).

[5] N. Burq and M. Hitrik, Energy decay for damped wave equations in partially rectangular domains, Math. Res. Let. 14 (2007), no. 1, 35-47.

[6] H. Christianson, private communication.

[7] - Corrigendum to Semiclassical non-concentration near hyperbolic orbits, J. Funct. Anal. 246 (2007), no. 2, 145-195.

[8] M. Hitrik, Eigenfrequencies and expansions for damped wave equations, Meth. Appl. Anal. 10 (2003), no. 4, 1-22.

[9] G. Lebeau, Équation des ondes amorties, Vol. 19 of Algebraic and geometric methods in mathematical physics, Kluwer Acad. Publ., Dordrecht (1993).

[10] S. Nonnenmacher and M. Zworski, Quantum decay rates in chaotic scattering, Acta Math. 203 (2009), no. 2, 149-233.

[11] Y. Pesin and B. Pitskel, Topological pressure and the variational principle for non-compact sets, Funct. Anal. and its Applications 18 (1984), no. 4, 50-63.

[12] Y. B. Pesin and V. Sadovskaya, Multifractal analysis and conformal Axiom A flows, Comm. Math. Phys. 216 (2001) 277-312.

[13] J. Rauch and M. Taylor, Decay of solutions to nondissipative hyperbolic systems on compact manifolds, Comm. in Pure and Appl. Anal. 28 (1975) 501-523.

[14] E. Schenck, Energy decay for the damped wave equation under a pressure condition, Comm. Math. Phys. 300 (2010), no. 2, 375-410.

[15] P. Walters, An introduction to ergodic theory, Vol. 79 of Graduate Texts in Mathematics, Springer (1975).

[16] - The variational principle for the pressure of continuous transformations, Amer. J. Math. 17 (1976) 937-971.

Mathematics Department, Northwestern University, 2033 Sheridan Road, Evanston IL 60208, USA.

E-mail address: schenck@math.northwestern.edu 